\title{
Temporal Expectation Modulates the Cortical Dynamics of Short-Term Memory
}

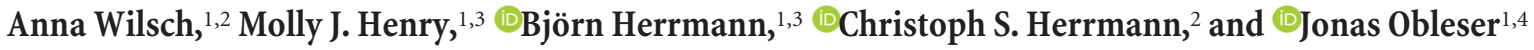 \\ ${ }^{1}$ Max Planck Research Group Auditory Cognition, Max Planck Institute for Human Cognitive and Brain Sciences, 04103 Leipzig, Germany, ${ }^{2}$ Experimental \\ Psychology Laboratory, Center for Excellence Hearing4all, European Medical School, University of Oldenburg, 26111 Oldenburg, Germany, ${ }^{3}$ Department of \\ Psychology, Brain and Mind Institute, University of Western Ontario, London, Ontario, N6A 3K7 Canada, and ${ }^{4}$ Department of Psychology, University of \\ Lübeck, 23562 Lübeck, Germany
}

Increased memory load is often signified by enhanced neural oscillatory power in the alpha range (8-13 Hz), which is taken to reflect inhibition of task-irrelevant brain regions. The corresponding neural correlates of memory decay, however, are not yet well understood. In the current study, we investigated auditory short-term memory decay in humans using a delayed matching-to-sample task with pure-tone sequences. First, in a behavioral experiment, we modeled memory performance over six different delay-phase durations. Second, in a MEG experiment, we assessed alpha-power modulations over three different delay-phase durations. In both experiments, the temporal expectation for the to-be-remembered sound was manipulated so that it was either temporally expected or not. In both studies, memory performance declined over time, but this decline was weaker when the onset time of the to-be-remembered sound was expected. Similarly, patterns of alpha power in and alpha-tuned connectivity between sensory cortices changed parametrically with delay duration (i.e., decrease in occipitoparietal regions, increase in temporal regions). Temporal expectation not only counteracted alpha-power decline in heteromodal brain areas (i.e., supramarginal gyrus), but also had a beneficial effect on memory decay, counteracting memory performance decline. Correspondingly, temporal expectation also boosted alpha connectivity within attention networks known to play an active role during memory maintenance. The present data show how patterns of alpha power orchestrate short-term memory decay and encourage a more nuanced perspective on alpha power across brain space and time beyond its inhibitory role.

Key words: alpha; attention; magnetoencephalography; neural oscillations; sensory memory; temporal expectations

\section{Significance Statement}

Our sensory memories of the physical world fade quickly. We show here that this decay of short-term memory can be counteracted by so-called temporal expectation; that is, knowledge of when to expect a sensory event that an individual must remember. We also show that neural oscillations in the "alpha" $(8-13 \mathrm{~Hz})$ range index both the degree of memory decay (for brief sound patterns) and the respective memory benefit from temporal expectation. Spatially distributed cortical patterns of alpha power show opposing effects in auditory versus visual sensory cortices. Moreover, alpha-tuned connectivity changes within supramodal attention networks reflect the allocation of neural resources as short-term memory representations fade.

\section{Introduction}

Short-term memory allows us to focus our attention on representations of perceptions that are no longer physically present (Bad-

\footnotetext{
Received Oct. 11, 2017; revised July 9, 2018; accepted July 10, 2018.

Author contributions: A.W., M.J.H., B.H., and J.O. designed research; A.W., M.J.H., B.H., and J.O. performed research; A.W. analyzed data; A.W., M.J.H., B.H., C.S.H., and J.0. wrote the paper.

This work was supported by the Max Planck Research Group (J.0.). We thank Burkhard Maess and Yvonne Wolff-Rosier for help with MEG data acquisition. Three anonymous reviewers helped improve the manuscript with their constructive comments.

The authors declare no competing financial interests.

Correspondence should be addressed to either Anna Wilsch or Jonas Obleser, Department of Psychology, Universität zu Lübeck, MFC 8, Maria-Goeppert-Straße 9a, 23562 Lübeck, Germany, E-mail: anna.wilsch@gmail.com or jonas.obleser@uni-luebeck.de.
}

deley, 2012). This ability is limited, though, by memory load and memory decay. The amount and the precision of information in memory draw on capacity and must not exceed a certain limit (Luck and Vogel, 1997; van den Berg et al., 2012; Ma et al., 2014; Joseph et al., 2016). Neural oscillations in the alpha range (8-13 $\mathrm{Hz}$ ) recorded using human EEG or MEG are modulated by manipulations of memory load. For example, alpha power increases as the number of items held in memory increases (Jensen et al., 2002; Busch and Herrmann, 2003; Leiberg et al., 2006; Obleser et al., 2012). This alpha-power increase is thought to protect the 
storage of items in memory (Roux and Uhlhaas, 2014) by functionally inhibiting task-irrelevant information and/or brain regions (Klimesch et al., 2007). However, it is less clear how neural oscillatory activity is related to memory decay.

Memory decay refers to fading away of the memory representation over time (Brown, 1958; Posner and Keele, 1967). Previous work on neural correlates of memory decay suggests a reduction of neural responses during the "delay phase"; that is, the time during which information is held in memory before it can be reported or compared with another stimulus. Over the course of memory delay, single-cell activity in monkey prefrontal cortex decreases (Fuster, 1999), as does the BOLD response measured in posterior (see Jha and McCarthy, 2000 for visual memory) and temporal (see Gaab et al., 2003 for auditory memory) cortical regions in humans.

It is less clear to predict how alpha power should behave over the course of a memory delay phase. Given the relationship between BOLD responses and cortical alpha power (Sadaghiani et al., 2010), alpha power might well decrease. However, if we assume that alpha oscillations inhibit interference with memory representations, then alpha power should increase over time because more interference is to be expected. In addition, the expected direction of alpha change with fading short-term memory should depend on the brain area under consideration: An auditory area in temporal cortex, where short-term memory traces need protection (Strauß et al., 2014), and a visual area, where interference needs to be inhibited (Jensen and Mazaheri, 2010), might well show differential response patterns.

One factor that has the potential to counteract memory decay is temporal expectation. Detection and discrimination are more accurate for temporally expected compared with unexpected stimuli (Coull and Nobre, 1998; Griffin et al., 2001; Nobre, 2001; Jaramillo and Zador, 2011) and temporally expected events promote perceptual evidence accumulation (Cravo et al., 2013). In addition, temporal expectation for a to-be-remembered stimulus reduces memory load for speech-in-noise, as indexed by improved memory performance (Wilsch et al., 2015). This load reduction co-occurred with decreased alpha power during stimulus maintenance, suggestive of temporal expectation effects persisting into the delay phase. Moreover, temporally expected distractors are more easily kept out of short-term memory. This effect is also accompanied by increased alpha power in anticipation of an expected distractor (Bonnefond and Jensen, 2012). It is unclear, however, whether temporal expectation also has a beneficial effect on memory decay (Kunert and Jongman, 2017).

Here, we report the results of two experiments investigating the time course of decay in short-term memory (Cowan, 1984; Cowan et al., 1997; Nees, 2016). Auditory short-term memory enables integration of auditory information and preservation of information over brief periods of time (Schröger, 2007). We conducted a delayed pitch comparison procedure (Harris, 1952; Bachem, 1954; Bull and Cuddy, 1972; Keller et al., 1995) with two brief pure-tone sequences embedded in noise separated by variable delay phases to investigate whether the sequences were the same as or different from each other.

Experiment 1 probed and modeled memory performance over six increasing delay phases addressing the question of whether temporal expectation affects memory decay behaviorally. Experiment 2 investigated the interaction of temporal expectation and memory decay at the neural level. The focus was on neural alpha $(\sim 8-13 \mathrm{~Hz})$ oscillatory dynamics during the maintenance of the expected (or not expected) stimulus as a remote effect of temporal expectation on memory decay. Alpha-power modulations were assessed on the sensor level as well as by means of source analyses and functional connectivity.

\section{Materials and Methods}

\section{Participants}

In Experiment 1 (behavior and modeling), $N=19$ healthy right-handed participants (12 females; age range 23-33 years, median 25 years) took part. In Experiment 2 (behavior and MEG recordings), an independent sample of $N=20$ healthy right-handed participants (10 females; age range 23-33 years, median 27 years) took part. All participants had selfreported normal hearing. The local ethics committee (University of Leipzig) approved of the studies in accordance with the Declaration of Helsinki. Participants were fully debriefed about the nature and goals of the studies and provided written informed consent before testing. All participants received financial compensation of $7 €$ per hour for their participation.

\section{Experimental task and stimuli}

The time course of an example trial is depicted in Figure 1A. On each trial, participants heard two pure-tone sequences (S1 and S2, see "Characteristics of sound stimuli" section) and responded whether they were the same or different. These pure-tone sequences were embedded in noise to increase perceptual load (Pichora-Fuller and Singh, 2006; van den Berg et al., 2012). Nonverbal stimuli were used to preclude rehearsal effects (Obleser and Eisner, 2009; Oberauer and Lewandowsky, 2013) and thus to keep any effects interpretable in terms of short-term memory.

Each trial began with the presentation of a fixation cross. After a brief pause (jittered between 0.75 and $1.25 \mathrm{~s}$ ), white noise and a visual cue were presented simultaneously. The visual cue indicated the onset time of the first sound (S1; see next paragraph) and remained on screen throughout the entire trial. Participants had to retain S1 in memory for a variable period of time. Then, a second sound (S2) was presented and participants made a "same" /"different" judgment by pressing one of two buttons on a response box. The response was prompted $\sim 1 \mathrm{~s}$ (jittered between $0.9 \mathrm{~s}$ and $1.1 \mathrm{~m}$ ) after the presentation of S2. Finally, participants indicated their confidence in their "same"/"different" response on a 3-level confidence scale ("not at all confident," "somewhat confident," or "very confident"). Trials were separated by an intertrial interval of $\sim 1 \mathrm{~s}$ (jittered between 0.75 and $1.25 \mathrm{~s}$ ) that was free of stimulation or responses. See Figure $1 A$ for an outline of a trial.

Operationalization of memory decay and temporal expectation. Memory decay was manipulated by varying the time interval (delay phase) between S1 and S2. The aim of Experiment 1 was to fit an exponential decay function to memory performance across different delay-phase durations. To this end, the delay-phase duration was varied logarithmically in six steps ranging between 0.6 and $7 \mathrm{~s}$ (i.e., 0.6, 1, 1.6, 2.6, 4.3, and $7 \mathrm{~s}$; Fig. $1 B$, left). In Experiment 2, delay phases were more coarsely sampled (1, 2, and $4 \mathrm{~s}$; Fig. $1 D$, left).

Temporal expectation for S1 was manipulated by varying the S1 onset times relative to the presentation of a visual cue. Onset times were either fixed (i.e., $\mathrm{S} 1$ occurred $1.3 \mathrm{~s}$ after the onset of the visual cue) or jittered (i.e., $\mathrm{S} 1$ occurred after a duration drawn from a uniform distribution ranging between $0.9 \mathrm{~s}$ and $1.7 \mathrm{~s}$, mean $=1.3 \mathrm{~s}$ ).

Characteristics of the sound stimuli. All sound stimuli were sequences consisting of five pure tones; each pure tone had a duration of $40 \mathrm{~ms}$, resulting in a total sound duration of $200 \mathrm{~ms}$ (Watson et al., 1975). Sound stimuli were presented in standard-deviant pairs. For the standard stimulus, the middle (third) tone's frequency was randomly selected on each trial from a uniform distribution ranging between 450 and $600 \mathrm{~Hz}$. The second and fourth tones were independently assigned frequencies $\pm 1-4$ semitones (ST) with respect to the frequency of the middle tone and the first and final tones were independently assigned frequencies $\pm 4-7$ ST with respect to the middle tone. Unique patterns were generated on each trial.

On half of the trials, a deviant stimulus was presented (i.e., "different" trials). For the deviant stimulus, the third and the fourth pure tone in the sequence were higher in frequency compared with S1. The third and fourth tones were both shifted up by the same amount (in ST; see "Pro- 
A Memory Decay

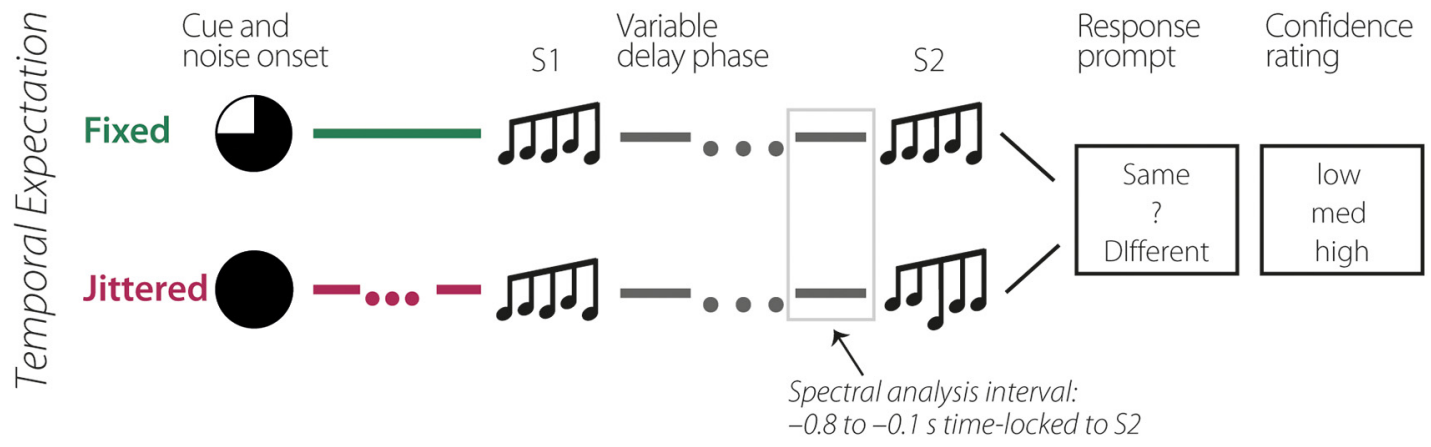

B

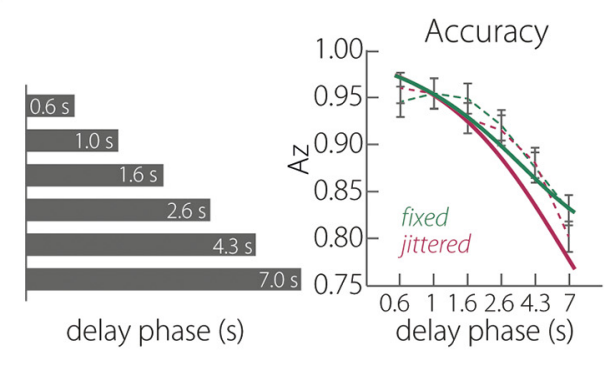

D

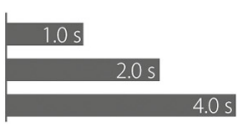

delay phase (s)

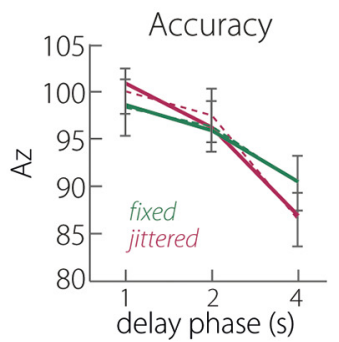

C

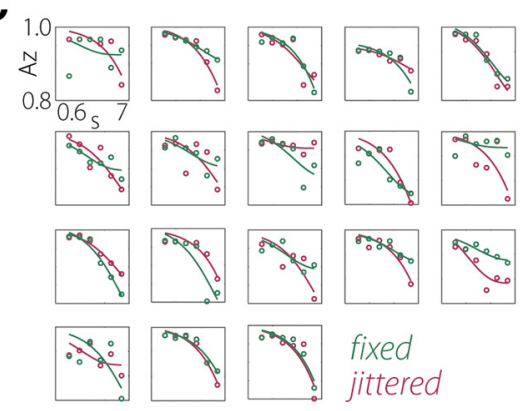

E

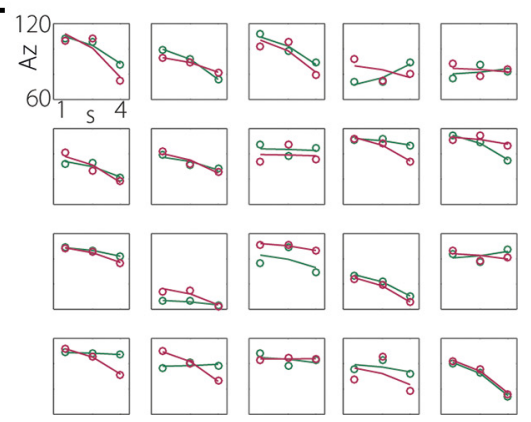

Figure 1. Experimental design and behavioral performance. $A$, Experimental design. The top illustrates a "same" trial (S1 and $S 2$ are the same) with a fixed onset time. The bottom illustrates a "different" trial (S1 and S2 are different) with jittered onset time. The actual durations of the variable delay phases are specified in $\boldsymbol{B}$ and $\boldsymbol{D}$. The light gray box indicates the interval of the spectral analyses: -0.8 to $-0.1 \mathrm{~s}$ time locked to S2. B, Memory performance in Experiment 1. The gray bars illustrate the six variable delay-phase durations from 0.6 to $7.0 \mathrm{~s}$ (i.e., values in each bar). The line graph displays averaged memory performance in Az (dotted lines) and the exponential fit (solid lines), both separately for fixed and jittered onset times; error bars indicate SEM of Az. The bar graphs show the average values for the estimated parameters "growth" and "decay," as well as the asymptote, separately for fixed and jittered onset times. Error bars display the SEM. In all graphs, green refers to fixed and magenta to jittered onset times. The asterisk indicates the significant difference between fixed and jittered onset times. $C$, Single-participant exponential fits. Every single plot displays the exponential fit of one participant separately for fixed (green) and jittered (magenta) onset times. Dots display the actual performance data Az. D, Memory performance in Experiment 2. The gray bars illustrate the three variable delay phase durations from 1.0 to $4.0 \mathrm{~s}$ (i.e., values in each bar). The line graph displays averaged memory performance in Az (RAU-transformed percentage; dotted lines) and the linear fit (solid lines) both separately for fixed and jittered onset times; error bars indicate SEM of Az. The bar graph shows the average values for the estimated slope separately for fixed and jittered onset times. Error bars display the SEM. In all graphs, green refers to fixed and magenta to jittered onset times. The asterisk indicates the significant difference between fixed and jittered onset times. $\boldsymbol{E}$, Single-participant linear fits. Every single plot displays the linear fit of one participant separately for fixed (green) and jittered (magenta) onset times. Note that the $x$-axis has been log-transformed and linear fits thus appear inflected. Dots display the actual performance data Az.

cedure" section). The exact standard-to-deviant-difference was adjusted for each participant individually (see "Procedure" section). Each pure tone had an onset and offset ramp of $10 \mathrm{~ms}$. On half of the trials, the standard stimulus was presented during the S1 interval, whereas the deviant stimulus was presented during the $\mathrm{S} 1$ interval the other half of the trials.

The noise masker was white noise. Sound sequences and noise were presented with a constant signal-to-noise ratio (SNR) of $-17 \mathrm{~dB}$. This SNR was determined via pilot testing to increase difficulty of the memory task but still allow all participants to perform the task.

Procedure

Before the MEG measurement, participants were familiarized with the stimuli and task and performed a few practice trials. Then, individual thresholds were estimated (i.e., the frequency difference between standard and deviant in the third and fourth pure tone position of the sound sequences). A custom adaptive-tracking procedure was used that yielded a frequency difference corresponding to memory performance falling between $65 \%$ and $85 \%$ correct responses.

In Experiment 1, participants completed 360 trials in 10 blocks of 36 trials each. In Experiment 2, brain activity was recorded with MEG during the performance of 396 trials completed in 12 blocks of 33 trials each. The manipulation of S1 onset time (fixed, jittered) was kept constant within a block and participants were informed at the start of each block about the type of temporal cue they would receive on each trial. Delayphase durations (0.6-7 s and 1, 2, and $4 \mathrm{~s}$, for Experiments 1 and 2, respectively) were equally distributed within blocks. The order of trials 
within a block and order of blocks were randomized for each participant. Button assignments were counterbalanced across participants such that half of the participants indicated that the first and the second sound were the same using the left button and half did so with the right button.

The testing took $\sim 2.5 \mathrm{~h}$ per participant and was conducted within one session. The overall session including practice blocks and preparation of the MEG setup took $\sim 4 \mathrm{~h}$.

\section{Modeling of behavioral data in Experiment 1}

Data analysis. The crucial measure for memory decay was the performance measure $\mathrm{Az}$, a nonparametric performance measure derived from confidence ratings. Hit and false alarm rates at each confidence level were used to construct receiver operating characteristic (ROC) curves (Macmillan and Creelman, 2004) for each condition and ROC curves were used to derive Az. Az corresponds to the area under the ROC curve and can be interpreted similarly to proportion correct. Az was computed for each of the 12 conditions (temporal expectation, $2, \times$ memory decay, 6 ), allowing us to estimate memory decay as a function of delay-phase duration separately for fixed and jittered onset times. One participant had to be excluded from this analysis because the participant did not make use of the entire confidence rating scale in at least two experimental conditions; Az could not be computed for these data points. Another participant presented the same behavior but only in one condition. Here, the missing Az value was interpolated by calculating the mean of the two adjacent conditions.

We fitted Equation 1 (Glass and Mackey, 1988) to Az scores as a function of delay-phase duration as follows:

$$
x(t)=x_{0}+e^{-\gamma t}+\frac{\lambda}{\gamma}\left(1-e^{-\gamma t}\right)
$$

where $t$ is equal to time (i.e., delay-phase duration) and $x_{0}$ corresponds to the intercept. This specific function contained a term describing decay, $\gamma$, and an additional term describing growth, $\lambda$. The ratio of $\lambda$ to $\gamma$ indicated the function's asymptote.

Note that, compared with simple decay functions (Wickelgren, 1969; Rubin and Wenzel, 1996), this function bears the advantage that it takes the nature of physiological systems into account. That is, it assumes that, in physiological systems, activations decline whereas competing, new activations arise. This interaction of decay and growth applies to shortterm memory in the following sense: The stored memory representation decays over time. According to so-called resource models of short-term memory, allocation of cognitive resources (i.e., growth of activation) can counteract this decay (for review, see Ma et al., 2014). Short-term memory has been argued to operate by effectively focusing attention on the memory representation (Cowan, 2000). This concept has been supported by findings of neural activity during the delay phase representing active storage mechanisms (Roux and Uhlhaas, 2014). Furthermore, prefrontal cortex activity has also been reported to be involved in active maintenance processes (Bauer and Fuster, 1976; Funahashi et al., 1993; for a review see D'Esposito, 2007).

The fit-initial parameters were as follows: $x_{0}=0, \gamma=0$, and $\lambda=0$, where $x_{0}$ was bound between 0 and 1 and $\gamma$ and $\lambda$ were bound between 0 and infinity. The model fit was computed with the lsqcurvefit function with MATLAB (version 8.2, Optimization Toolbox; The MathWorks), which allowed for 1000 iterations.

In addition, we also fitted a decay-term-only model; that is, the first term: $x(t)=\left(x_{0}+e^{-y t}\right)$. The decay-only model is more parsimonious and more commonly used to estimate memory decay (Peterson and Peterson, 1959; Wickelgren, 1969). To determine which one of these two models represented the memory performance data best, we calculated the Bayesian information criterion (BIC; Schwarz, 1978) for both model fits, as well as for fixed and jittered onset times separately. Note that the BIC penalizes for more parameters and allows for an equitable comparison of goodness-of-fit of both models (smaller is better). We averaged the BICs across fixed and jittered onset times separately for each function. 17 of 18 participants had a lower BIC for the full model (Eq. 1) than the decay-only model, indicating an overall better fit by the former model. Therefore, all further analyses were conducted on the parameters resulting from the fit of the complete Equation 1. Four of the participants were excluded from subsequent analyses because $R^{2}$, an indicator for goodness of the model fit, of their fitted models was smaller than 0.3 (see Fig. $1 C$ for individual model fits). The average $R^{2}$ values for the fixed and jittered conditions, respectively, were $0.66(S D=0.31 ; 0.80, S D=0.13$ without excluded participants) and $0.72(\mathrm{SD}=0.25 ; 0.81, \mathrm{SD}=0.16$ without excluded participants).

After the fitting of the function, the resulting parameters $x_{0}, \gamma$, and $\lambda$ for jittered and fixed onset times as dependent variables were assessed with a multivariate ANOVA. This allowed us to test whether there was a global difference between jittered and fixed onset times. Subsequently, the parameters $x_{0}, \gamma$, and $\lambda$ were tested for differences between fixed and jittered onset times with univariate repeated-measures ANOVAs to determine whether memory decay was less strong when $\mathrm{S} 1$ onset times were predictable.

\section{Data recording and analysis in Experiment 2}

Participants were seated in an electromagnetically shielded room (Vacuumschmelze). Magnetic fields were recorded using a 306-sensor Neuromag Vectorview MEG (Elekta) with 204 orthogonal planar gradiometers and 102 magnetometers at 102 locations. Two electrode pairs recorded a bipolar EOG for horizontal and vertical eye movements. The participants' head positions were monitored during the measurement by five head position indicator (HPI) coils. Signals were sampled at a rate of $1000 \mathrm{~Hz}$ with a bandwidth ranging from direct current (DC) to $330 \mathrm{~Hz}$.

The signal space separation method was applied offline to suppress external interferences in the data, interpolate bad channels, and transform individual data to a default head position that allows statistical analyses across participants in sensor space (Taulu et al., 2004).

Subsequent data analyses were carried out with MATLAB and the FieldTrip toolbox (Oostenveld et al., 2011) using only trials to which correct responses were provided ("correct trials"). Analyses were conducted using only the 204 gradiometer sensors because they are most sensitive to magnetic fields originating directly underneath them (Hämäläinen et al., 1993). The continuous data were filtered offline with a $0.5 \mathrm{~Hz}$ high-pass filter specifically designed to provide a strong suppression of DC signals in the data ( $>140 \mathrm{~dB}$ at DC, 3493 points, Hamming window; Ruhnau et al., 2012).

Subsequently, trial epochs ranging from -1.5 to $11.5 \mathrm{~s}$ time locked to the onset of S1 were defined. The use of long epochs prevented windowing artifacts in the time-frequency analysis; the intervals analyzed statistically were shorter (see below). Epochs were low-pass filtered at $80 \mathrm{~Hz}$ and subsequently down-sampled to $200 \mathrm{~Hz}$.

Epochs with strong artifacts were rejected when the signal range at any gradiometer exceeded $800 \mathrm{pT} / \mathrm{m}$. Independent component analysis (ICA) was applied to the epochs to reduce artifacts due to eye blinks and heartbeat. After ICA, remaining epochs were rejected when the signal range within one epoch exceeded $200 \mathrm{pT} / \mathrm{m}$ (gradiometer) or $100 \mu \mathrm{V}$ (EOG). Additionally, trials were rejected manually for which variance across sensors was deemed high relative to all others (per participant, per condition) based on visual inspection. For further analysis, each trial was time locked at two different points; that is, all trials were time locked to the first stimulus ( $t=0 \mathrm{~s}$ at $S 1$ onset) and to the second stimulus ( $t=0 \mathrm{~s}$ at $\mathrm{S} 2$ onset) for separate analyses. This was because different trials had different delay phase durations so that trials time locked to S1 were not always time locked to S2.

\section{Spectral analysis}

The analyses focus on responses time locked to S2. This enabled us to examine the responses related to the end of the delay phase, the period during which we expected to find remote effects of temporal expectation for $\mathrm{S} 1$ on stimulus maintenance and thus on memory decay. For each trial, a $0.7 \mathrm{~s}$ segment was extracted ( -0.8 to $-0.1 \mathrm{~s}$ time locked to S2 excluding evoked responses due to $\mathrm{S} 1$ sound presentation; Fig. $1 A$, light gray box), multiplied with a Hann taper, and the power between 8 and 13 $\mathrm{Hz}$ was computed using a fast Fourier transform (FFT).

For illustration purposes only, we also computed time-frequency representations (TFRs) of responses that were time locked to S1. Timefrequency analysis was conducted on trial epochs ranging from -2.0 to 
$7.6 \mathrm{~s}$ for each trial (with $20 \mathrm{~ms}$ time resolution) for frequencies ranging from 0.5 to $20 \mathrm{~Hz}$ (logarithmically spaced in 20 bins). Single-trial timedomain data were convolved with a Hann taper with an adaptive width of 2-4 cycles per frequency (i.e., 2 cycles for $0.5-1.6 \mathrm{~Hz}, 3$ cycles for $1.9-9.2$ $\mathrm{Hz}$, and 4 cycles for $11.1-20 \mathrm{~Hz}$ ). The output of the analysis was complex Fourier data. For further analyses, power (squared magnitude of the complex-valued TFR estimates) was averaged across single trials. Intertrial phase coherence (ITPC) was computed based on the complex Fourier data (Lachaux et al., 1999). ITPC is the magnitude of the amplitude-normalized complex values averaged across trials for each time-frequency bin per channel and experimental condition (Thorne et al., 2011).

Next, FFT power spectra and TFRs were averaged across gradiometers in each pair. This procedure resulted in one single-trial value for each time point (TFRs only), frequency bin, and sensor position for each delay-phase condition and onset-time condition.

\section{Source localization}

To estimate the origin of sensor-level alpha-power, source localizations were computed based on individual T1-weighted MRI images (3T Magnetom Trio; Siemens). Topographical representations of the cortical surface of each hemisphere were constructed with Freesurfer (http://surfer. nmr.mgh.harvard.edu) and the MR coordinate system was coregistered with the MEG coordinate system using the HPIs and $\sim 100$ additional digitized points on the head surface (Polhemus FASTRAK 3D digitizer). For forward and inverse calculations, boundary element models were computed for each participant using the inner skull surface as volume conductor (using the MNE toolbox; https://martinos.org/mne/). Individual mid-gray matter surfaces were used as source model by reducing the $\sim 150,000$ vertices needed to describe single hemispheres to 10,242 vertices.

The beamformer approach, dynamic imaging of coherent sources; Gross et al., 2001) was used to project alpha power ( -0.8 to $-0.1 \mathrm{~s}$ time locked to S2-onset) to source space. To this end, a multitaper FFT centered at $11 \mathrm{~Hz}$ ( $\pm 2 \mathrm{~Hz}$ smoothing with three Slepian tapers; Percival and Walden, 1993) was computed. A complex filter was calculated based on the data of all delay-phase and onset-time conditions (Gross et al., 2001; Schoffelen et al., 2008). Single-trial complex FFT data were then projected through the filter, separately for each condition providing a power value for each frequency bin in the alpha range at each vertex.

Functional connectivity analyses. To attain a better understanding of the functional role of alpha power in memory decay, specifically for alpha power emerging from left superior temporal gyrus (STG, MNI $[-50$, $-17,-8])$, connectivity analyses between cortical sources were computed. Note that MNI coordinates were selected based on the source localization of the alpha power effect (see below). A whole-brain approach was adopted to find brain areas that were functionally connected with left STG on the basis of weighted pairwise phase consistency (wPPC; Vinck et al., 2010; see also Gulbinaite et al., 2017). The critical advantage of wPPC over, for example, intertrial phase coherence, is that wPPC is independent of the number of trials used in the calculation. wPPC measures the consistency of phase angles between trial pairs. First, Fourier spectra from 8 to $13 \mathrm{~Hz}$ were calculated in the time window time locked to $\mathrm{S} 2(-0.8$ to $-0.1 \mathrm{~s})$ and multiplied by the previously calculated common DICS filter (see above). Then, wPPC was computed using the FieldTrip function ft_connectivity_ppc.m on the single-trial complex Fourier spectra. The greater the wPPC at a vertex, the greater the phase consistency between this vertex and left STG.

\section{Statistical analysis}

Memory performance. Analogous to Experiment 1, memory performance for each condition was indexed by Az (Fig. 1D). To test whether there were differences in Az between experimental conditions (i.e., fixed and jittered onset times and delay phase duration), we computed a repeatedmeasures ANOVA with both factors temporal expectation (fixed vs jittered onset times) and memory decay $(1,2$, and $4 \mathrm{~s}$ delay-phase duration). In analogy to Experiment 1, we also assessed the difference in memory decay between fixed and jittered onset times. Because, in Experiment 2, only three different delay-phase durations were used instead of six, we were only able to compute a linear fit across these durations.
Therefore, memory decay was estimated by regressing Az on the delay phase durations of 1,2 , and $4 \mathrm{~s}$. The impact of temporal expectation on memory decay was measured by comparing the slopes of the linear fit for fixed and jittered S1-onset times using a paired-samples $t$ test. Before all analyses, $\mathrm{Az}$ was linearized by computing a rationalized arcsine unit (RAU) transformation (Studebaker, 1985). Furthermore, as effect size measures, we report partial $\eta^{2}$ for repeated-measures ANOVAs and $r_{\text {equivalent }}$ for dependent-samples $t$ tests (Rosenthal and Rubin, 2003). Response times are not reported because responses were cue prompted and thus would provide only partially valid information about costs and benefits of the experimental manipulations.

Sensor level analyses. Statistical analyses were conducted on the FFT power spectra ( -0.8 to -0.1 time locked to S2) according to a multilevel approach. On the first (single-subject) level, we regressed alpha power on the delay-phase durations $(1,2$, and 4 s) similar to the regression of memory performance $(\mathrm{Az})$ on delay-phase duration (see above). To test the parametric modulation of memory decay, the FieldTrip-implemented independent-samples regression $t$ test was performed (Maris and Oostenveld, 2007). The regression $t$ test provides the regression $b$ coefficient (i.e., slope of the modulation) for each frequency bin at each of the 102 sensor positions indicating the strength of the tested contrast. Here, to test for a linear relationship between alpha power and delay phase duration, contrast coefficients were selected corresponding to the actual delay-phase duration in seconds (i.e., 1, 2, and 4). To test whether temporal expectation had an impact on this relationship, the same contrast was calculated for fixed and jittered onset times separately.

For the statistical analyses on the second (group) level, $b$-values resulting from the first-level statistics testing the parametric modulations of alpha power by the delay phase were tested against zero. In addition, to test whether the delay-phase modulation in the fixed condition differs significantly from the modulation in the jittered condition, $b$-values attained for each of the onset-time conditions separately were tested against each other. The tests against zero as well as the tests contrasting fixed and jittered conditions were conducted with FieldTrip's dependent-samples $t$ test using cluster-based permutation tests. The cluster test corrects for multiple comparisons resulting from testing each frequencysensor combination. All cluster tests were two-tailed and were thus considered significant when $p<0.025$.

We also tested for correlations between alpha power and memory performance $(\mathrm{Az})$, averaging over experimental conditions, with a multilevel cluster test. On the first level, each participant's six RAUtransformed Az-values (2 temporal-expectancy conditions $\times 3$ delay phases) were correlated with the corresponding alpha-power values. On the second level, first-level correlation values were Fisher's $z$ transformed and tested against zero with a dependent-samples cluster-based permutation $t$ test.

Source level analyses. Statistical analyses for source-projected alpha power as well as for wPPC reflecting functional connectivity between left STG and any other vertex were conducted with the same approach. The aim was to test whether either variable (alpha power or wPPC) was modulated by delay-phase duration and if this modulation was affected by temporal expectation.

Contrasts were calculated for each vertex separately. To test for a linear relationship of memory decay and alpha power in source space, source projected alpha power and the delay-phase duration $(1,2$, and $4 \mathrm{~s})$ were $z$-transformed on a single-subject level. Then, the delay phase duration served as a regressor and was fitted to the source power to test for a linear relationship of alpha power and delay-phase duration. The same approach was applied to test for effects of functional connectivity: wPPCs were $z$-transformed and $z$-transformed delay-phase duration values were fitted to these wPPCs.

The resulting regression coefficients at each individual vertex from both contrasts were then spatially smoothed across the surface (vertices) using an approximation to a $6 \mathrm{~mm}$ FWHM Gaussian kernel (Han et al., 2006) and morphed onto a common surface in MNI space, respectively (Freesurfer average brain; Fischl et al., 1999). For the interaction of temporal expectation and memory decay, the same linear regression was applied to the same data again but separately for each temporalexpectation condition. On the group level, regression coefficients of each 
contrast were tested against zero or fixed-onset-time coefficients were tested against jittered-onset-time coefficients, respectively, with vertexwise $t$ tests. The resulting $t$-values were $z$-transformed and displayed on the average brain surface with contrast dependent uncorrected vertexwise threshold of $|z| \geq 1.96$ (Sohoglu et al., 2012).

Then, brain regions that showed statistical effects were identified by extracting the MNI coordinate of the greatest $z$-value within one area of interest. Areas of interest were identified by visual inspection. The MNI coordinate was then used to identify the specific brain region using the MNI structural atlas.

Correlation of alpha power and Az in source space. Analogous to the analyses on the sensor level, the correlation of source-projected alpha power and $\mathrm{Az}$ was calculated by correlating RAU-transformed Az with alpha power within condition at each vertex point. Here as well, the Fisher's $z$-transformed correlation values were tested against zero with vertexwise $t$ tests. The resulting $t$-values were $z$-transformed and displayed on the average brain surface with an uncorrected vertexwise threshold of $|z| \geq 1.96$.

\section{Results}

In the present study, we investigated whether and how temporal expectation ameliorates the decay of sound representations in short-term memory. Participants retained a sound (S1) in memory for a delay phase that varied in duration from trial to trial and judged whether sound S1 was same or different from another sound (S2) presented after the delay phase. We focused on behavioral performance as well as on neural oscillatory activity in the alpha frequency band.

\section{Experiment 1: Behavioral modeling of memory decay}

In Experiment 1, we estimated a "forgetting curve" based on fits of an exponential-decay function to Az values as a function of delay-phase duration. Fits were conducted separately for fixed and jittered S1-onset times to assess the effect of temporal expectation on memory decay. Consistent with the broad literature on short-term memory decay, Az declined with longer delay-phase durations. Interestingly, performance decayed differently for jittered and fixed onset times (Fig. 1B). The two functions (jittered and fixed) show that, for delay phases up to $1 \mathrm{~s}$, memory performance was the same following fixed and jittered onset times, whereas for longer delay phases, performance declined less severely following fixed compared with jittered onset times. Figure $1 C$ displays the single-subject fits of the decay function.

A multivariate ANOVA showed that the estimated parameters decay factor, growth factor, and intercept (Wilk's approximated $F_{(3,11)}=3.81, p=0.043, \eta^{2}=0.51$ ) differed for fixed versus jittered S1-onset times. Subsequent univariate tests on all parameters separately revealed that there was a trend-level effect of the decay factor $\gamma\left(F_{(1,13)}=3.68, p=0.077, \eta^{2}=0.221\right.$; Fig. $\left.1 B\right)$. The univariate test on the growth factor $\lambda$ showed that growth over delay-phase duration was significantly greater for fixed than for jittered onset times $\left(F_{(1,13)}=4.95, p=0.044, \eta^{2}=0.276\right.$; Fig. $1 B)$, converging with the test on the decay factor $\gamma$ that Az declines faster after jittered than after fixed onset times. The univariate test on the intercept $x_{0}$ did not show a difference between onset times $\left(F_{(1,13)}=0.04, p=0.84, \eta^{2}=0.003\right)$. Next, we tested both asymptotes separately against 0.5 (i.e., corresponding to memory performance at chance level). The asymptote parameter estimate corresponding to fixed onset times was significantly larger than chance as shown by a $95 \%$ confidence interval (CI) of $0.52-0.82$, whereas the asymptote after jittered onset times did not differ from 0.5 (95\% CI $=0.26-0.64$; Fig. $1 B)$. However, fixed and jittered asymptotes did not differ significantly from each other $\left(t_{(13)}=1.47, p=0.164, r=0.378\right)$. Therefore, whereas
A Grand average power and inter-trial phase coherence

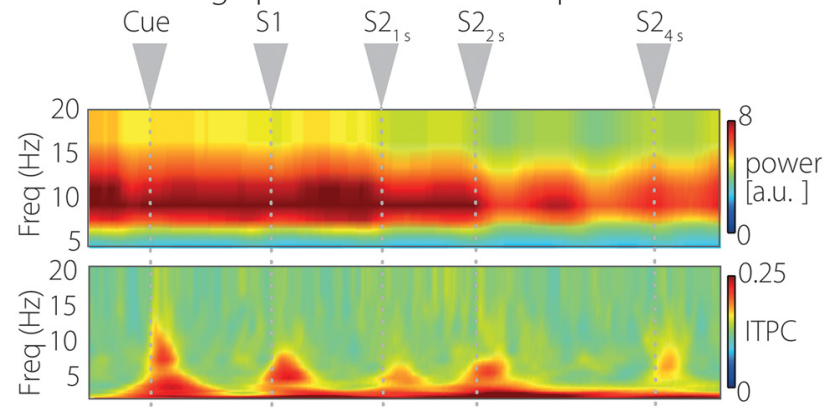

B Grand average al pha power

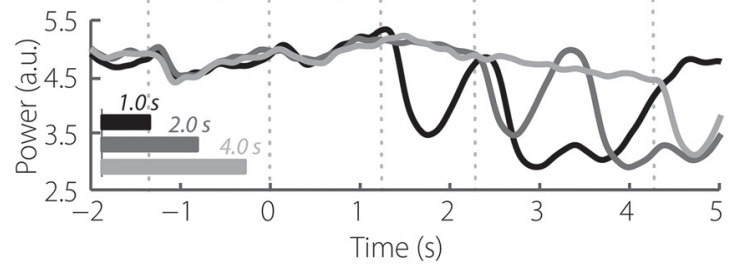

Figure 2. Time-frequency grand averages power and phase coherence. $\boldsymbol{A}$, Top, Grandaverage power $5-20 \mathrm{~Hz}$ averaged across all sensors. Gray arrows on top indicate stimulus occurrence times. $\$ 1$ refers to the to-be-remembered stimulus. $\$ 2$ refers to the second stimulus. The index indicates the corresponding delay-phase duration in seconds. Bottom, Grand average of intertrial phase coherence $5-20 \mathrm{~Hz}$ averaged across all sensors. B, Alpha power $(8-13 \mathrm{~Hz})$ grand-average across channels per delay-phase duration.

memory performance declines to chance level following jittered onset times for longer delays, fixed onset times counteract this decline in memory performance.

\section{Experiment 2: Linear effects of memory decay and temporal expectation on behavioral performance}

First, we computed a repeated-measures ANOVA on Az with the factors delay-phase duration and temporal expectation. The ANOVA on RAU-transformed Az yielded a significant main effect of delay-phase duration $\left(F_{(2,38)}=21.95, p<0.0001, \eta^{2}=\right.$ $0.536)$ but no main effect of temporal expectation $\left(F_{(1,19)}=3.20\right.$, $\left.p=0.736, \eta^{2}=0.1441\right)$. The interaction of both factors $\left(F_{(2,38)}=\right.$ 28.76, $\left.p=0.048, \eta^{2}=0.602\right)$ followed up by post hoc $t$ tests revealed that $\mathrm{Az}$ after $1 \mathrm{~s}$ and $2 \mathrm{~s}$ delay-phase duration did not differ by temporal expectation $\left(1 \mathrm{~s}: t_{(19)}=-1.19, p=0.248, r=\right.$ $\left.0.263 ; 2 \mathrm{s:} t_{(19)}=-0.64, p=0.528, r=0.145\right)$. However, after a 4 s delay-phase duration, participants performed significantly worse when onset times had been jittered compared with fixed onset times $\left(t_{(19)}=2.24, p=0.037, r=0.457\right)$. In addition, we performed single-subject linear fits on fixed and jittered memory decline over time. Consistent with the findings of Experiment 1, the comparison of the single-subject slopes for fixed and jittered onset times revealed that short-term memory performance (as indicated $\mathrm{Az}$ ) after jittered onset times decayed faster than after fixed onset times $\left(t_{(19)}=2.51, p=0.0213, r=0.499\right.$, Fig. $1 D$; for single-subject linear fits, see Fig. $1 E$; note that the $x$-axis has been log-transformed and linear fits thus appear inflected).

\section{Experiment 2: Effects of memory decay and temporal expectation on alpha power}

We were interested in how memory decay was affected by temporal expectation and how this relationship would relate to alpha-power modulation. Figure $2 A$ (top) illustrates overall power for all frequency bands $(5-20 \mathrm{~Hz})$ time locked to the onset of S1 (averaged across all channels). Figure $2 B$ presents the time 
A Memory decay effect
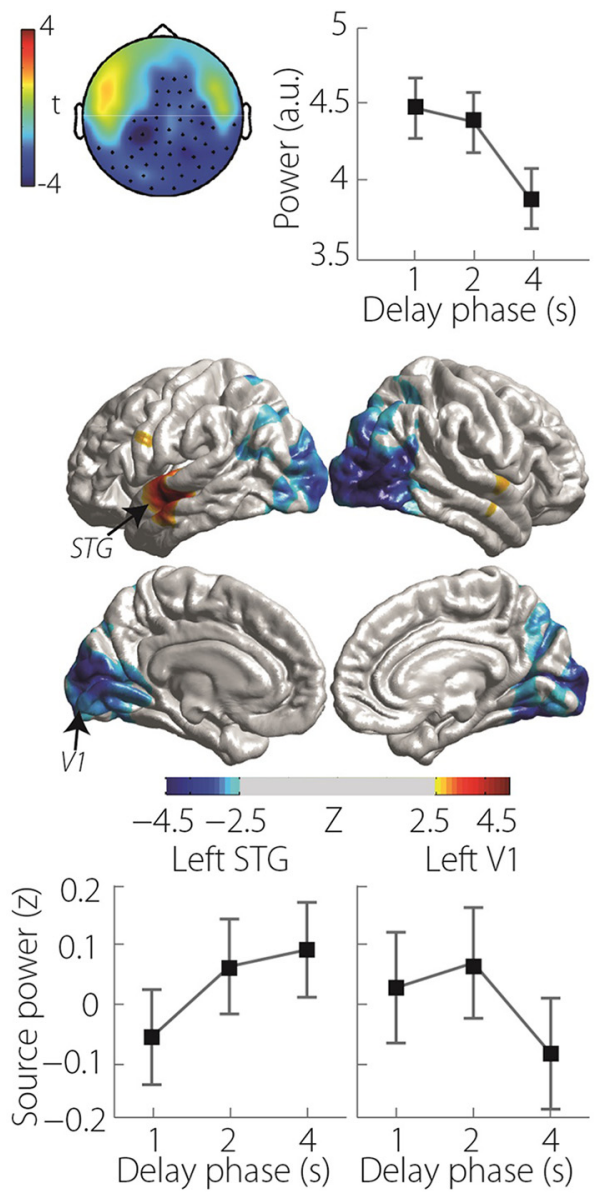

B Temporal expectations $\times$ Memory decay
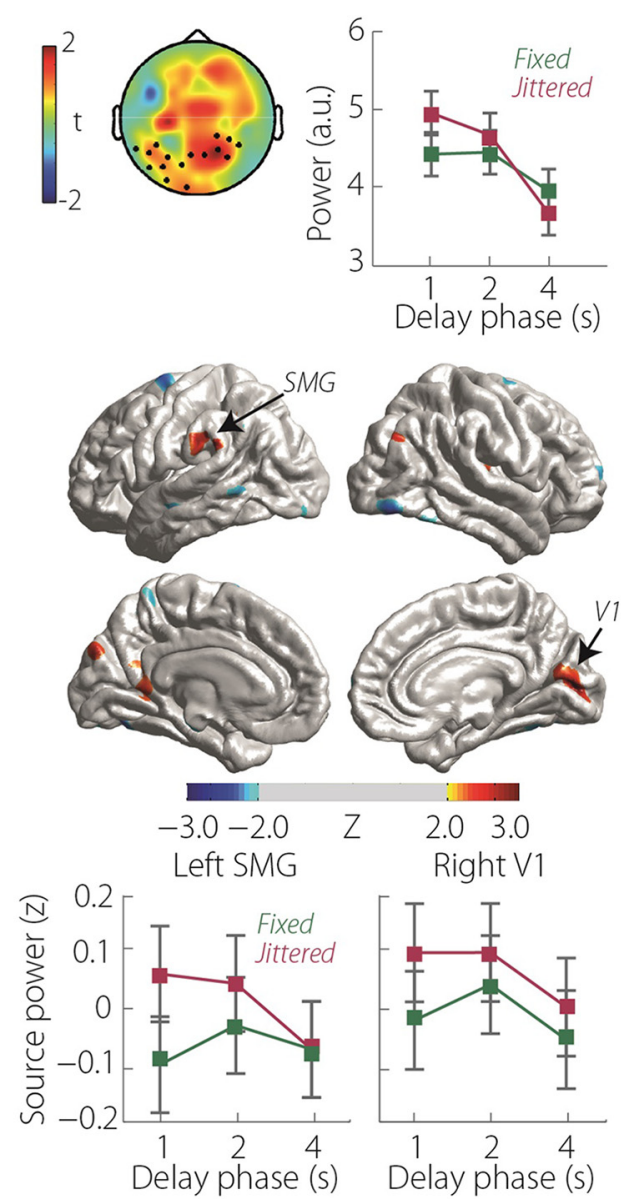

Figure 3. Condition effects in alpha power. $\boldsymbol{A}$, Effect of memory decay (1,2, and $4 \mathrm{~s}$ delay phase). Top, Topographies of the $t$-values of the linear fit of alpha power on delay-phase duration on the sensor level. Marked channels present the significant cluster. The line graph represents alpha power extracted from the displayed channels. Bottom, Source projected linear fit of alpha power on delay-phase duration. $z$-transformed $t$-values are displayed with a threshold of $|z| \geq 1.96$. Line graphs display delay-phase activity drawn from and averaged across the vertices presenting peak activity around left STG and left V1. All error bars show within-subject SE. B, Impact of temporal expectation on memory decay. Top, Topographies of the $t$-values of the impact of onset-time condition on the linear fit of alpha power on delay-phase duration on the sensor level. Marked channels present significant cluster. Line graphs represent alpha power extracted from the displayed channels. Bottom, Source projected difference between fixed and jittered onset times of the linear fit of alpha power on delay-phase duration. $z$-transformed $t$-values are displayed with a threshold of $|z| \geq$ 1.96. Positive $z$-values indicate that jittered onset times have a steeper slope than fixed onset times. Line graphs display condition-wise activity drawn from and averaged across the vertices presenting peak activity around left SMG and right V1. All error bars display within-subject SE.

course of alpha power averaged across trials for each condition separately. Following S1, alpha power increases until the earliest occurrence of S2 (i.e., shortest delay phase of $1 \mathrm{~s}$ ) and then decreases slowly. ITPC (Fig. 2A, bottom) is increased time locked to the visual cue and the auditory events. Apart from the cue-related response, the ITPC peak frequency is below the alpha range for sound-related responses. Below, we will focus on alpha power.

We investigated alpha-power changes as a function of delay phase ( -0.8 to $-0.1 \mathrm{~s}$ time locked to S2; cf. Fig. 1$)$ and whether the relationship between delay-phase duration and alpha power was modulated by temporal expectation.

The first-level $b$ coefficients resulting from the linear regression of alpha power on delay-phase duration were tested against zero on the group level. $b$ coefficients were significantly smaller than zero in a broad posterior, negative cluster $(p<0.0001$; Fig. $3 A$, top), indicating that alpha-power decreased with longer delay-phase duration. A second cluster test contrasting the $b$ coefficients of the fixed-onset-time condition with the $b$ coefficients of the jittered-onset-time condition showed that temporal expectation also had an impact on alpha power: alpha power decreased less with increasing delay-phase durations following fixed onset times compared with jittered onset times (left-posterior positive cluster, $p=0.025$; Fig. 3B, top).

\section{Source localization of alpha-power modulations}

Source localizations were computed to identify the brain regions underlying the reported alpha-power effects on the sensor level. The effect of delay-phase duration on alpha power localized to occipital and temporal sites. The negative peak indicating a decrease of alpha power with increasing delay-phase duration emerged from left primary visual cortex (V1 MNI: $-5,-88,11)$. In addition to the negative cluster (retrieved from sensor level analysis), source localization revealed a positive linear relationship between alpha power and delay-phase duration emerging from left STG (MNI: $-50,-17,-8)$. $z$-transformed effects in source space and $z$-values $>1.96$ for each delay-phase condition averaged across vertices around the peak effect in left V1 and left STG are illustrated in line graphs of Figure $3 A$ (bottom).

The differential effect of temporal expectation on alpha power during the delay phase originated most prominently from left 


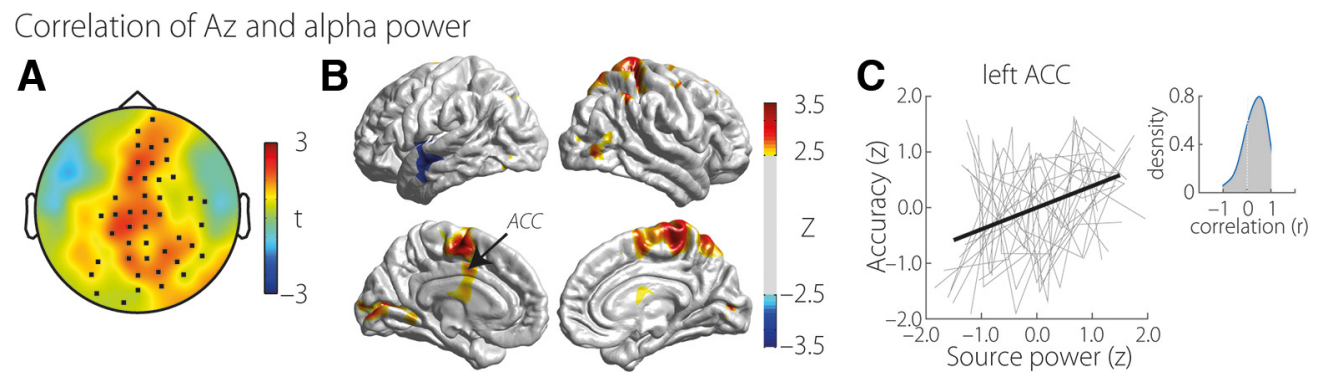

Figure 4. Correlation of sensitivity in memory performance (Az) and alpha power. $A$, Topography of the correlation of alpha power and $A z$ ( $t$-values). Black dots display channels that belong to the significant positive cluster. $\boldsymbol{B}$, Alpha power emerging from highlighted brain areas correlates with Az. Positive z-values indicate a positive correlation of Az and alpha power. $\boldsymbol{C}$, Relation of anterior cingulate cortex $(\mathrm{ACC})$ alpha power to accuracy. The gray lines show the single-subject z-transformed raw data of alpha power in left ACC and Az. The black line indicates the grand average correlation. The blue line of the inlet displays the density of the correlation values across all participants.

supramarginal gyrus (SMG MNI: $-54,-37,32)$ and right V1 (MNI: 14, -80, 13) (Fig. 3B, bottom). In these brain regions, alpha power was higher with longer delays when temporal expectation was present.

\section{Alpha power predicts behavioral performance}

In a final analysis, we aimed to relate the observed modulation of memory performance (i.e., Az) to alpha-power modulations. We correlated Az with alpha power, across all conditions, by means of a cluster test, which revealed a centrally distributed positive cluster ( $p=0.022$; Fig. $4 A$ ). Figure $4 B$ illustrates the source projections of the correlation effect and Figure $4 C$ displays the singlesubject correlations between $\mathrm{Az}$ and source alpha drawn from left anterior cingulate cortex (ACC). During the delay phase, the positive correlation of alpha power and Az emerged from left ACC (MNI: $-2,2,38$ ), bilateral postcentral gyrus (MNI: 28, -34, 70; MNI: $-4,-9,56$ ), and bilateral occipital cortices (MNI: 7, -64, 62; MNI: $-7,-86,2)$. A negative correlation between alpha power and Az emerged from left STG (MNI: - 55, - 10, - 37).

Note that the positive (V1) as well as negative (STG) correlations of alpha power and performance in this particular analysis are most likely due to the common, confounding variable of delay-phase duration itself, as these regions were identified before to correlate negatively and positively with delay phase duration, respectively.

Figure $4 C$ displays the linear relation of alpha power in left ACC and single-subject in short-term memory performance ac$\operatorname{curacy}(\mathrm{Az})$.

\section{Functional connectivity with left STG}

Source projections of alpha power revealed a pattern of brain regions susceptible to memory decay. Most prominent effects originated from left STG and bilateral visual cortices. To attain a better understanding of the functional role of alpha power and its different origins, we computed functional connectivity in the alpha range. Due to the strong alpha power effect in left STG (Fig. $3 A$ ) as well as its crucial role in auditory short-term memory (Sabri et al., 2004), left STG was used as a seed in a whole-brain connectivity analysis. The aim of this analysis was to find brain regions that were functionally connected with left STG and where this connectivity was modulated by memory decay and temporal expectation.

Connectivity analyses revealed that, with longer delay-phase duration, phase consistency between left STG and bilateral inferior frontal gyrus (IFG; left MNI: -32, 3, 14; right MNI: 52, 32, $-9)$ as well as left premotor cortex (MNI: $-6,-12,63)$ increased. In contrast, left-STG connectivity with left primary visual cortex (V1; MNI: 26, -100, -10) and right STG (MNI: 65,
-16, 6) decreased (Fig. 4A). Moreover, some memory-decayrelated changes in connectivity were modulated by temporal expectation: In right SMG (MNI: $62,-35,40)$ as well as in right hippocampus (MNI: 15, -37, 9) connectivity with left STG increased with delay-phase duration after fixed onset times and decreased after jittered onset times (Fig. 4B).

To attain a better understanding of the role of functional wPPC-based connectivity with left STG for memory performance, we tested whether inter-individual differences in connectivity between STG and right V1, as well as between STG and left IFG (i.e., the areas of the decay main effect; Fig. 4), led to interindividual differences in memory performance (i.e., Az).

We split the group of participants into high- and lowconnectivity groups (median split) for V1 as well as for IFG. Then, we compared performance, as indexed by Az, between high- and low-connectivity participants using separate mixedmeasures ANOVAs (with delay-phase duration 1, 2, and $4 \mathrm{~s}$ as a within-subject factor) for V1 and IFG connectivity. For V1, ANOVA revealed a significant main effect of delay phase duration $\left(F_{(2,32)}=18.44, p<0.0001, \eta^{2}=0.535\right)$ and a significant main effect of high versus low connectivity $\left(F_{(1,16)}=5.22, p=\right.$ $0.036, \eta^{2}=0.246$; Fig. 5). For IFG, the main effect of high versus low connectivity as well as all interactions did not turn out to be significant (all $F<1.39$; Fig. 5). The same analysis on the interaction effect in SMG and hippocampus did not yield any significant relationship between degree of functional connectivity and memory performance (i.e., Az).

In sum, these results demonstrate that overall higher alphafrequency functional connectivity between STG and V1 is beneficial for short-term memory performance.

\section{Discussion}

In two experiments, we investigated how decay in auditory shortterm memory interacts with temporal expectation and alpha power. The data demonstrate that memory decay can be partially counteracted by temporal expectation. That is, decay is attenuated when the onset time of to-be-remembered items is fixed (and therefore highly predictable) compared with when the onsets are jittered. Second, we observed a potential trading relation between the power of alpha oscillations generated in visual and auditory regions, such that increases of alpha with delay phase were observed in auditory cortices, whereas decreases were observed in visual cortices. We also observed attenuation of alpha-power modulations by temporal expectation, paralleling memory performance, in functional networks beyond auditory cortex, namely primary visual cortex, bilateral supramarginal gyrus, and hippocampal regions. 

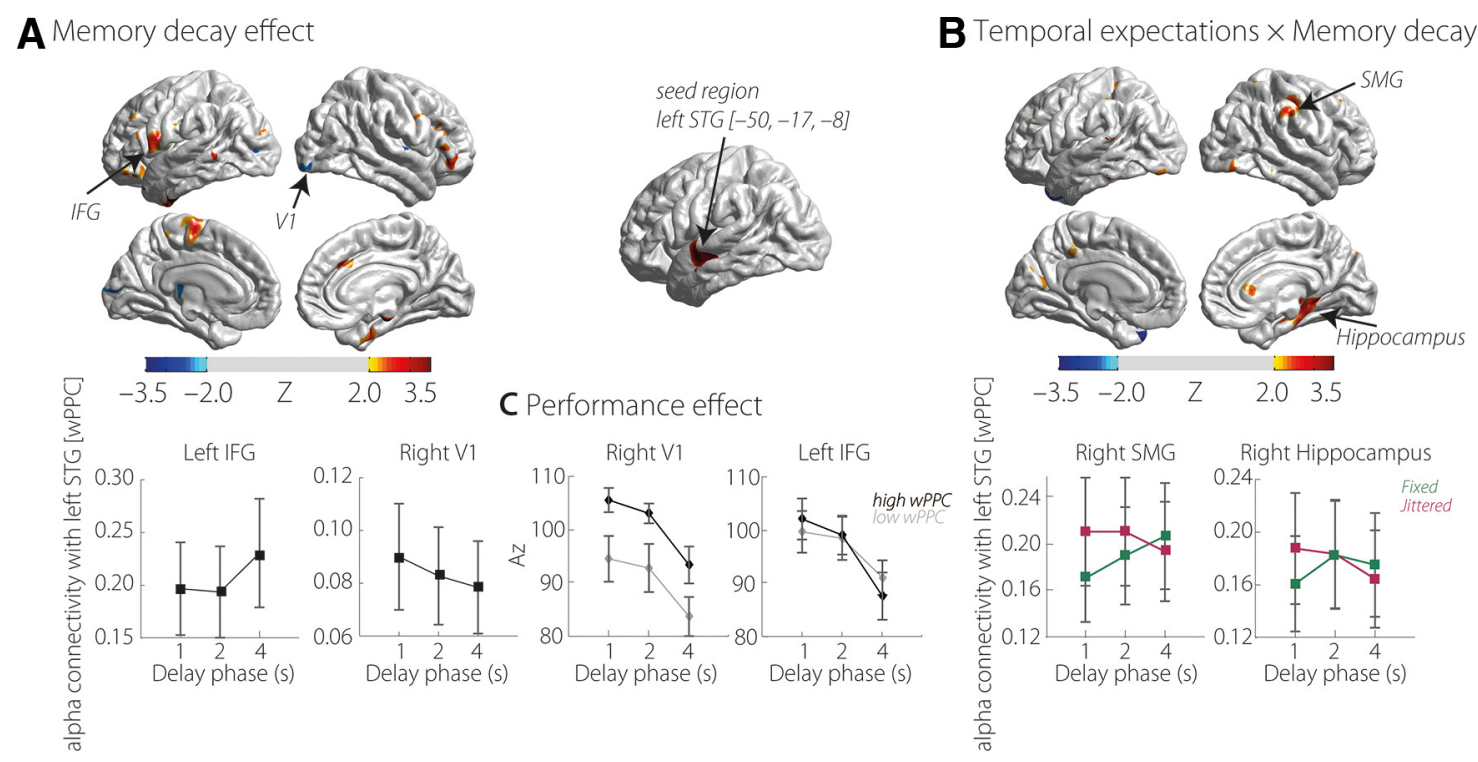

Figure 5. Functional connectivity. $A$, Effect of memory decay $(1,2$, and $4 \mathrm{~s}$ delay phase). Functional connectivity of left STG and highlighted brain areas is modulated by delay phase duration. $z$-transformed $t$-values are displayed with a threshold of $|z| \geq 1.96$. Positive $z$-values describe an increase of the phase locking value with delay-phase duration; negative $z$-values indicate a decrease of phase locking with delay-phase duration. Line graphs display the phase locking value between left STG and right V1 and left IFG, respectively, for each delay-phase duration. Error bars represent within-subject SE. B, Effect of temporal expectation on memory decay. Differential impact of fixed and jittered onset times on phase locking of left STG and highlighted brain areas along different delay phases. $z$-transformed $t$-values are displayed with a threshold of $|z| \geq 1.96$. Positive $z$-values indicate that the slope of the correlation of phase locking and delay-phase duration is greater after fixed onset times than after jittered onset times. Negative z-values indicate that this correlation has a greater slope after jittered than after fixed onset times. Line graphs display the phase-locking value between left STG and right SMG and right hippocampus, respectively, for each delay-phase duration and each onset time condition (green line displays fixed and red line displays jittered onset times). Error bars represent within-subject SE. The brain topography in the center illustrates the seed region (i.e., left STG) of the connectivity analysis. C, Effect of alpha connectivity on memory performance. Both plots show memory performance for low and high alpha connectivity (wPPC) between left STG and right V1 (left plot) and left IFG (right plot) for each delay-phase duration. Black lines represent performance after high connectivity; gray lines indicate performance after low connectivity. Error bars indicate SEM.

\section{Behavioral modeling of memory decay reveals benefit from temporal expectation}

In both studies, we were able to replicate the well established finding that, the longer an item is stored in short-term memory, the poorer the memory performance (Posner and Keele, 1967; Cowan et al., 1997). This can be explained by a "fading away" of the memory representation over time (Brown, 1958).

Critically, both experiments show that the decline of memory performance over time (decay) can be counteracted by temporal expectation. Performance was better when the onset time of the to-be-remembered sound was perfectly predictable compared with when it was jittered. Using an exponential decay function to model behavioral performance (Experiment 1) revealed that the growth factor was increased. We argue here that this factor reflects allocated cognitive resources that counteract the fading away of the memory representation (i.e., decay).

Previous work suggests that prior knowledge about the timeof-occurrence of the to-be-remembered item enhances encoding precision during stimulus presentation (Rohenkohl et al., 2012), which in turn enables the maintenance of the item in memory for a longer period. Another (not mutually exclusive) framework, the time-based resource-sharing model (Barrouillet et al., 2004, 2007; Barrouillet and Camos, 2012), suggests that a memory trace requires attention to be maintained and that the trace decays over time as the attentional focus moves away from the representation. The higher the memory load, the fewer attentional resources are available for memory maintenance (Ma et al., 2014). These interpretations cannot be distinguished based on the analyses performed here. However, we suggest a hybrid model in which enhanced encoding precision (due to temporal expectation) frees attentional resources by reducing memory load (Wilsch et al., 2015) and consequently facilitates stimulus maintenance over time.

\section{Differential alpha modulations beyond auditory cortex underlie short-term memory and its decay}

Alpha power during retention was modulated parametrically by delay-phase duration. Consistent with a decline in memory performance, alpha power decreased over time in bilateral primary visual cortex. Alpha-power decreases during memory-delay phases have been reported to emerge from occipitoparietal brain regions (Krause et al., 1996; Jensen et al., 2002; Jokisch and Jensen, 2007; Tuladhar et al., 2007; Sauseng et al., 2009; Haegens et al., 2010; Bonnefond and Jensen, 2012; Wöstmann et al., 2015). Classically, occipitoparietal alpha power during auditory memory tasks is interpreted as reflecting inhibition of visual areas so that resources can be allocated to maintenance of auditory information.

In contrast, in left temporal cortex (i.e., STG encompassing primary auditory cortex), alpha power increased with longer memory-delay times. Based on previous fMRI studies, STG supports active stimulus maintenance during auditory short-term memory (Sabri et al., 2004; Grimault et al., 2009; Kumar et al., 2016). In general, activity in sensory cortices is associated with the maintenance of memory representations (for review on visual short-term memory, see Sreenivasan et al., 2014; for review on auditory cortex activity, see Linke and Cusack, 2015).

Alpha power has been argued to protect this storage of items in memory (Roux and Uhlhaas, 2014). Corroborating this view, an auditory-memory retroactive-cueing paradigm recently demonstrated increased alpha power in a network including STG after presentation of a retro cue that allowed the participant to select an object from memory and prioritize it (Lim et al., 2015). Therefore, we tentatively suggest that the observed alpha-power increase reflects the allocation of attentional resources needed to prevent the fading away of the memory representation over time, rather than inhibitory mechanisms as are classically associated 
with occipitoparietal alpha. The dissociation between alpha's behavior in visual and auditory cortices supports the presence of distributed alpha systems in the brain, supporting at least partly different functions (Başar et al., 1997).

Finally, with increasing delay-phase duration, the alpha-band functional connectivity between left STG and left IFG as well as between left STG and left premotor cortex increased, whereas such connectivity between the left STG and contralateral right V1 as well as between left STG and contralateral right STG decreased. With respect to the former finding, IFG and premotor cortex have both been assigned storage roles in auditory short-term memory (Koelsch et al., 2009). IFG has been shown to be actively involved in phonological maintenance (Paulesu et al., 1993; Awh et al., 1996; Kumar et al., 2016), especially when auditory information cannot be rehearsed, as for tonal stimuli (Gruber and von Cramon, 2003) or in the present setup.

Kumar et al. (2016) also observed increased functional connectivity between auditory cortex and left IFG during the maintenance of single tones in memory. They argue that this connectivity is part of a system of auditory short-term memory maintaining sound-specific representations by projections from higher-order areas, such as rehearsal of pitch (Koelsch et al., 2009). Therefore, the presently observed increased connectivity between STG with IFG and premotor cortex would reflect alphatuned, active top-down modulations of STG.

In contrast, the decreased connectivity between left STG and left V1 goes well with the overall decrease of alpha power in V1 with longer delay-phase durations. If this decline in alpha power does indeed reflect a decline in functional inhibition, as argued above (Jensen et al., 2002), we suggest that, due to this decline in connectivity, irrelevant information emerging from visual cortex is less inhibited and more likely to interfere with the memory representation held active in STG. We see a functional relevance of this connectivity for optimal memory maintenance: Across participants, overall higher connectivity between STG and V1 was associated with better memory performance (Fig. 5).

\section{Benefit from temporal expectation emerges from heteromodal brain areas}

One primary goal of the experiments presented here was to determine whether temporal expectation influences memory decay and the accompanying alpha power modulations. In fact, in left SMG and in V1, alpha power declined faster following jittered compared with fixed onset times (i.e., behaved similar to memory performance). $\mathrm{V} 1$ alpha power arguably inhibits irrelevant information such as interfering visual input. However, the alphapower decline there is less strong after fixed onset times, possibly indicating enhanced inhibition of irrelevant visual processing over time.

The SMG has previously been observed to be crucial for stimulus maintenance in auditory short-term memory (see Paulesu et al., 1993; van Dijk et al., 2010; Obleser et al., 2012 for increased alpha power in SMG during auditory short-term memory). For example, Lim et al. (2015) found alpha power in SMG to be increased after a valid attention-guiding retro-cue compared with a neutral cue while maintaining a syllable in auditory shortterm memory. In their study as well as in the present study, alpha power was increased when memory maintenance was facilitated due to an attentional cue. Furthermore, Gaab et al. (2003) investigated pitch memory with fMRI and identified bilateral SMG to be a short-term pitch-information storage site. The BOLD signal emerging from the left SMG correlated positively with perfor- mance at the pitch-memory task, underlining the active role of left SMG for auditory short-term memory.

We also observed differential effects of fixed versus jittered sound onsets on alpha connectivity from left STG to right SMG and right hippocampus. Increased connectivity under high temporal expectation further demonstrates the crucial role of $S M G$ for active stimulus maintenance. Hippocampus, considered a vital part of long-term memory (Jeneson and Squire, 2012), has been found also to play a crucial role in short-term memory maintenance (Graham et al., 2010; Kumar et al., 2016). Underlining the importance of hippocampus for auditory short-term memory, Kumar et al. (2016) demonstrated increased connectivity of right hippocampus and auditory cortex during auditory short-term memory maintenance in particular. Therefore, the increase in alpha connectivity of left STG with both areas, SMG and hippocampus, most likely reflects increased neural resource allocation that prevents memory representations from fading away.

Last, we tested with a brain-wide analysis for correlations of alpha power and short-term memory performance. A positive correlation in anterior cingulate cortex replicated previous findings that increased alpha power is beneficial for short-term memory or short-term memory performance (Haegens et al., 2010; Roux et al., 2012; Lim et al., 2015; Wilsch et al., 2015). The anterior cingulate cortex, part of the cingulo-opercular network, is crucial for top-down control (for review, see Dosenbach et al., 2007, 2008; Petersen and Posner, 2012). In this view, alpha power provides a task-beneficial "steering rhythm" in and across the relevant top-down attention and sensory networks (Pinal et al., 2015).

\section{Implications of alpha power for auditory short-term memory} Overall, the present data demonstrate how alpha power serves as a proxy for the degree of decay in short-term memory. However, the brain region in which alpha modulations are observed, as well as the direction of alpha-power changes, informs us regarding the role of alpha oscillations generated in different neural networks. Aligning our alpha-power findings with our modeling analysis of memory performance, we tentatively suggest that increased temporal alpha power after temporally expected stimuli reflects the allocation of additional resources that refresh the representation maintained in memory (Lim et al., 2015; Wilsch and Obleser, 2016). The present data show that the mechanisms by which alpha power impacts on behavioral outcomes are complex and are hardly captured by a singular mechanism such as functional inhibition. All findings shown here, however, are compatible with a view of alpha-power as a modulatory, top-down signal (Kayser et al., 2015; Sedley et al., 2016; Wöstmann et al., 2017) that can help structure neural signaling. The present findings altogether encourage a more nuanced perspective on alpha power and its inhibitory role across brain areas and (trial) time. Most importantly, we could demonstrate that temporal expectation can alleviate memory decay, as reflected in memory performance and concomitant alpha-power modulations.

\section{References}

Awh E, Jonides J, Smith E, Schumacher E, Koeppe RA, Katz S (1996) Dissociation of storage and rehearsal in verbal working memory: evidence from positron emission tomography. Psychol Sci 7:25-31. CrossRef

Bachem A (1954) Time factors in relative and absolute pitch determination. J Acoust Soc Am 26:751-753. CrossRef

Baddeley A (2012) Working memory: theories, models, and controversies. Annu Rev Psychol 63:1-29. CrossRef Medline 
Barrouillet P, Camos V (2012) As time goes by: temporal constraints in working memory. Curr Dir Psychol Sci 21:413-419. CrossRef

Barrouillet P, Bernardin S, Camos V (2004) Time constraints and resource sharing in adults' working memory spans. J Exp Psychol Gen 133:83-100. CrossRef Medline

Barrouillet P, Bernardin S, Portrat S, Vergauwe E, Camos V (2007) Time and cognitive load in working memory. J Exp Psychol Learn Mem Cogn 33:570-585. CrossRef Medline

Başar E, Schürmann M, Başar-Eroglu C, Karakaş S (1997) Alpha oscillations in brain functioning: an integrative theory. Int J Psychophysiol 26:5-29. CrossRef Medline

Bauer RH, Fuster JM (1976) Delayed-matching and delayed-response deficit from cooling dorsolateral prefrontal cortex in monkeys. J Comp Physiol Psychol 90:293-302. CrossRef Medline

Bonnefond M, Jensen O (2012) Alpha oscillations serve to protect working memory maintenance against anticipated distracters. Curr Biol 22:19691974. CrossRef Medline

Brown J (1958) Some tests of the decay theory of immediate memory. Q J Exp Psychol 10:12-21. CrossRef

Bull AR, Cuddy LL (1972) Recognition memory for pitch of fixed and roving stimulus tones. Percept Psychophys 11:105-109. CrossRef

Busch NA, Herrmann CS (2003) Object-load and feature-load modulate EEG in a short-term memory task. Neuroreport 14:1721-1724. CrossRef Medline

Coull JT, Nobre AC (1998) Where and when to pay attention: the neural systems for directing attention to spatial locations and to time intervals as revealed by both PET and fMRI. J Neurosci 18:7426-7435. CrossRef Medline

Cowan N (1984) On short and long auditory stores. Psychol Bull 96:341370. CrossRef Medline

Cowan N (2000) Processing limits of selective attention and working memory: Potential implications for interpreting. Interpreting 5:117-146. CrossRef

Cowan N, Saults JS, Nugent LD (1997) The role of absolute and relative amounts of time in forgetting within immediate memory: the case of tone-pitch comparisons. Psychon Bull Rev 4:393-397. CrossRef

Cravo AM, Rohenkohl G, Wyart V, Nobre AC (2013) Temporal expectation enhances contrast sensitivity by phase entrainment of low-frequency oscillations in visual cortex. J Neurosci 33:4002-4010. CrossRef Medline

D’Esposito M (2007) From cognitive to neural models of working memory. Philos Trans R Soc B Biol Sci 362:761-772. CrossRef Medline

Dosenbach NU, Fair DA, Miezin FM, Cohen AL, Wenger KK, Dosenbach RA, Fox MD, Snyder AZ, Vincent JL, Raichle ME, Schlaggar BL, Petersen SE (2007) Distinct brain networks for adaptive and stable task control in humans. Proc Natl Acad Sci U S A 104:11073-11078. CrossRef Medline

Dosenbach NU, Fair DA, Cohen AL, Schlaggar BL, Petersen SE (2008) A dual-networks architecture of top-down control. Trends Cogn Sci 12:99105. CrossRef Medline

Funahashi S, Bruce CJ, Goldman-Rakic PS (1993) Dorsolateral prefrontal lesions and oculomotor delayed-response performance: evidence for mnemonic "scotomas". J Neurosci 13:1479-1497. CrossRef Medline

Fischl B, Sereno MI, Tootell RB, Dale a M (1999) High-resolution intersubject averaging and a coordinate system for the cortical surface. Hum Brain Mapp 8:272-284. CrossRef

Fuster JM (1999) Memory in the cerebral cortex: an empirical approach to neural networks in the human and nonhuman primate. Cambridge, MA: MIT.

Gaab N, Gaser C, Zaehle T, Jancke L, Schlaug G (2003) Functional anatomy of pitch memory-an fMRI study with sparse temporal sampling. Neuroimage 19:1417-1426. CrossRef Medline

Glass L, Mackey M (1988) From clocks to chaos: the rhythms of life. Princeton, NJ: Princeton University.

Graham KS, Barense MD, Lee AC (2010) Going beyond LTM in the MTL: a synthesis of neuropsychological and neuroimaging findings on the role of the medial temporal lobe in memory and perception. Neuropsychologia 48:831-853. CrossRef Medline

Griffin IC, Miniussi C, Nobre AC (2001) Orienting attention in time. Front Biosci 6:D660-D671. Medline

Grimault S, Lefebvre C, Vachon F, Peretz I, Zatorre R, Robitaille N, Jolicoeur P (2009) Load-dependent brain activity related to acoustic short-term memory for pitch: magnetoencephalography and fMRI. Ann N Y Acad Sci 1169:273-277. CrossRef Medline
Gross J, Kujala J, Hamalainen M, Timmermann L, Schnitzler A, Salmelin R (2001) Dynamic imaging of coherent sources: studying neural interactions in the human brain. Proc Natl Acad Sci U S A 98:694-699. CrossRef Medline

Gruber O, von Cramon DY (2003) The functional neuroanatomy of human working memory revisited: evidence from 3-T fMRI studies using classical domain-specific interference tasks. Neuroimage 19:797-809. CrossRef Medline

Gulbinaite R, Illhan B, VanRullen R (2017) The triple-flash illusion reveals a driving role of alpha-band reverberations in visual perception. J Neurosci 37:7219-7230. CrossRef Medline

Haegens S, Osipova D, Oostenveld R, Jensen O (2010) Somatosensory working memory performance in humans depends on both engagement and disengagement of regions in a distributed network. Hum Brain Mapp 31:26-35. CrossRef Medline

Hämäläinen M, Hari R, Ilmoniemi RJ, Knuutila J, Lounasmaa O (1993) Magnetoencephalography-theory, instrumentation, and applications to noninvasive studies of the working human brain. Rev Mod Phys 65:413497. CrossRef

Han X, Jovicich J, Salat D, van der Kouwe A, Quinn B, Czanner S, Busa E, Pacheco J, Albert M, Killiany R, Maguire P, Rosas D, Makris N, Dale A, Dickerson B, Fischl B (2006) Reliability of MRI-derived measurements of human cerebral cortical thickness: the effects of field strength, scanner upgrade and manufacturer. Neuroimage 32:180-194. CrossRef Medline

Harris JD (1952) The decline of pitch discrimination with time. J Exp Psychol 43:96-99. CrossRef Medline

Jaramillo S, Zador AM (2011) The auditory cortex mediates the perceptual effects of acoustic temporal expectation. Nat Neurosci 14:246-251. CrossRef Medline

Jeneson A, Squire LR (2012) Working memory, long-term memory, and medial temporal lobe function. Learn Mem 19:15-25. CrossRef Medline

Jensen O, Mazaheri A (2010) Shaping functional architecture by oscillatory alpha activity: gating by inhibition. Front Hum Neurosci 4:186. CrossRef Medline

Jensen O, Gelfand J, Kounios J, Lisman JE (2002) Oscillations in the alpha band $(9-12 \mathrm{~Hz})$ increase with memory load during retention in a shortterm memory task. Cereb Cortex 12:877-882. CrossRef Medline

Jha AP, McCarthy G (2000) The influence of memory load upon delayinterval activity in a working-memory task: an event-related functional MRI study. J Cogn Neurosci 12:90-105. CrossRef Medline

Jokisch D, Jensen O (2007) Modulation of gamma and alpha activity during a working memory task engaging the dorsal or ventral stream. J Neurosci 27:3244-3251. CrossRef Medline

Joseph S, Teki S, Kumar S, Husain M, Griffiths TD (2016) Resource allocation models of auditory working memory. Brain Res 1640:183-192. CrossRef Medline

Kayser C, Wilson C, Safaai H, Sakata S, Panzeri S (2015) Rhythmic auditory cortex activity at multiple timescales shapes stimulus-response gain and background firing. J Neurosci 35:7750-7762. CrossRef Medline

Keller TA, Cowan N, Saults JS (1995) Can auditory memory for tone pitch be rehearsed? J Exp Psychol Learn Mem Cogn 21:635-645. CrossRef Medline

Klimesch W, Sauseng P, Hanslmayr S (2007) EEG alpha oscillations: the inhibition-timing hypothesis. Brain Res Rev 53:63-88. CrossRef Medline

Koelsch S, Schulze K, Sammler D, Fritz T, Müller K, Gruber O (2009) Functional architecture of verbal and tonal working memory: an fMRI study. Hum Brain Mapp 30:859-873. CrossRef Medline

Krause CM, Lang AH, Laine M, Kuusisto M, Pörn B (1996) Event-related. EEG desynchronization and synchronization during an auditory memory task. Electroencephalogr Clin Neurophysiol 98:319-326. CrossRef Medline

Kumar S, Joseph S, Gander PE, Barascud N, Halpern AR, Griffiths TD (2016) A brain system for auditory working memory. J Neurosci 36:4492-4505. CrossRef Medline

Kunert R, Jongman SR (2017) Entrainment to an auditory signal: is attention involved? J Exp Psychol Gen 146:77-88. CrossRef Medline

Lachaux JP, Rodriguez E, Martinerie J, Varela FJ (1999) Measuring phase synchrony in brain signals. Hum Brain Mapp 8:194-208. CrossRef Medline

Leiberg S, Lutzenberger W, Kaiser J (2006) Effects of memory load on cortical oscillatory activity during auditory pattern working memory. Brain Res 1120:131-140. CrossRef Medline 
Lim SJ, Wöstmann M, Obleser J (2015) Selective attention to auditory memory neurally enhances perceptual precision. J Neurosci 35:1609416104. CrossRef Medline

Linke AC, Cusack R (2015) Flexible information coding in human auditory cortex during perception, imagery, and STM of complex sounds. J Cogn Neurosci 27:1322-1333. CrossRef Medline

Luck SJ, Vogel EK (1997) The capacity of visual working memory for features and conjunctions. Nature 390:279-281. CrossRef Medline

Ma WJ, Husain M, Bays PM (2014) Changing concepts of working memory. Nat Neurosci 17:347-356. CrossRef Medline

Macmillan NA, Creelman CD (2004) Detection theory: a user's guide, Ed 2. Mahwah, NJ: Lawrence Erlbaum Associates.

Maris E, Oostenveld R (2007) Nonparametric statistical testing of EEG- and MEG-data. J Neurosci Methods 164:177-190. CrossRef Medline

Nees MA (2016) Have we forgotten auditory sensory memory? Retention intervals in studies of nonverbal auditory working memory. Front Psychol 7:1892. CrossRef Medline

Nobre AC (2001) Orienting attention to instants in time. Neuropsychologia 39:1317-1328. CrossRef Medline

Oberauer K, Lewandowsky S (2013) Evidence against decay in verbal working memory. J Exp Psychol Gen 142:380-411. CrossRef Medline

Obleser J, Eisner F (2009) Pre-lexical abstraction of speech in the auditory cortex. Trends Cogn Sci 13:14-19. CrossRef Medline

Obleser J, Wöstmann M, Hellbernd N, Wilsch A, Maess B (2012) Adverse listening conditions and memory load drive a common alpha oscillatory network. J Neurosci 32:12376-12383. CrossRef Medline

Oostenveld R, Fries P, Maris E, Schoffelen JM (2011) FieldTrip: open source software for advanced analysis of MEG, EEG, and invasive electrophysiological data. Comput Intell Neurosci 2011:156869. CrossRef Medline

Paulesu E, Frith CD, Frackowiak RS (1993) The neural correlates of the verbal component of working memory. Nature 362:342-345. CrossRef Medline

Percival D, Walden A (1993) Spectral analysis for physical applications: multitaper and conventional univariate techniques. Cambridge: Cambridge University.

Petersen SE, Posner MI (2012) The attention system of the human brain: 20 years after. Annu Rev Neurosci 35:73-89. CrossRef Medline

Peterson LR, Peterson MJ (1959) Short-term retention of individual verbal items. J Exp Psychol 58:193-198. CrossRef Medline

Pichora-Fuller MK, Singh G (2006) Effects of age on auditory and cognitive processing: implications for hearing aid fitting and audiologic rehabilitation. Trends Amplif 10:29-59. CrossRef Medline

Pinal D, Zurrón M, Díaz F, Sauseng P (2015) Stuck in default mode: inefficient cross-frequency synchronization may lead to age-related short-term memory decline. Neurobiol Aging 36:1611-1618. CrossRef Medline

Posner MI, Keele SW (1967) Decay of visual information from a single letter. Science 158:137-139. CrossRef Medline

Rohenkohl G, Cravo AM, Wyart V, Nobre AC (2012) Temporal expectation improves the quality of sensory information. J Neurosci 32:8424-8428. CrossRef Medline

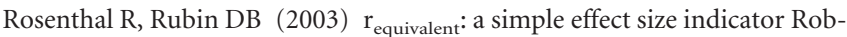
ert. Psychol Methods 8:492-496. CrossRef Medline

Roux F, Uhlhaas PJ (2014) Working memory and neural oscillations: alphagamma versus theta-gamma codes for distinct WM information? Trends Cogn Sci 18:16-25. CrossRef Medline

Roux F, Wibral M, Mohr HM, Singer W, Uhlhaas PJ (2012) Gamma-band activity in human prefrontal cortex codes for the number of relevant items maintained in working memory. J Neurosci 32:12411-12420. CrossRef Medline

Rubin D, Wenzel A (1996) One hundred years of forgetting: a quantitative description of retention. Psychol Rev 103:734-760. CrossRef

Ruhnau P, Herrmann B, Schröger E (2012) Finding the right control: the mismatch negativity under investigation. Clin Neurophysiol 123:507512. CrossRef Medline

Sabri M, Kareken DA, Dzemidzic M, Lowe MJ, Melara RD (2004) Neural correlates of auditory sensory memory and automatic change detection. Neuroimage 21:69-74. CrossRef Medline
Sadaghiani S, Scheeringa R, Lehongre K, Morillon B, Giraud AL, Kleinschmidt A (2010) Intrinsic connectivity networks, alpha oscillations, and tonic alertness: a simultaneous electroencephalography/functional magnetic resonance imaging study. J Neurosci 30:10243-10250. CrossRef Medline

Sauseng P, Klimesch W, Heise KF, Gruber WR, Holz E, Karim AA, Glennon M, Gerloff C, Birbaumer N, Hummel FC (2009) Brain oscillatory substrates of visual short-term memory capacity. Curr Biol 19:1846-1852. CrossRef Medline

Schoffelen JM, Oostenveld R, Fries P (2008) Imaging the human motor system's beta-band synchronization during isometric contraction. Neuroimage 41:437-447. CrossRef Medline

Schröger E (2007) Mismatch negativity. J Psychophysiol 21:138-146. CrossRef

Schwarz G (1978) Estimating the dimension of a model. Ann Stat 6:461464. CrossRef

Sedley W, Gander PE, Kumar S, Kovach CK, Oya H, Kawasaki H, Howard MA, Griffiths TD (2016) Neural signatures of perceptual inference. Elife 5:e11476. CrossRef Medline

Sohoglu E, Peelle JE, Carlyon RP, Davis MH (2012) Predictive top-down integration of prior knowledge during speech perception. J Neurosci 32: 8443-8453. CrossRef Medline

Sreenivasan KK, Curtis CE, D'Esposito M (2014) Revisiting the role of persistent neural activity during working memory. Trends Cogn Sci 18:8289. CrossRef Medline

Strauß A, Wöstmann M, Obleser J (2014) Cortical alpha oscillations as a tool for auditory selective inhibition. Front Hum Neurosci 8:350, CrossRef Medline

Studebaker GA (1985) A "rationalized" arcsine transform. J Speech Lang Hear Res 28:455-462. CrossRef

Taulu S, Kajola M, Simola J (2004) Suppression of interference and artifacts by the signal space separation method. Brain Topogr 16:269-275. Medline

Thorne JD, De Vos M, Viola FC, Debener S (2011) Cross-modal phase reset predicts auditory task performance in humans. J Neurosci 31:3853-3861. CrossRef Medline

Tuladhar AM, ter Huurne N, Schoffelen JM, Maris E, Oostenveld R, Jensen O (2007) Parieto-occipital sources account for the increase in alpha activity with working memory load. Hum Brain Mapp 28:785-792. CrossRef Medline

van den Berg R, Shin H, Chou WC, George R, Ma WJ (2012) Variability in encoding precision accounts for visual short-term memory limitations. Proc Natl Acad Sci U S A 109:8780-8785. CrossRef Medline

van Dijk H, Nieuwenhuis IL, Jensen O (2010) Left temporal alpha band activity increases during working memory retention of pitches. Eur J Neurosci 31:1701-1707. CrossRef Medline

Vinck M, van Wingerden M, Womelsdorf T, Fries P, Pennartz CM (2010) The pairwise phase consistency: a bias-free measure of rhythmic neuronal synchronization. Neuroimage 51:112-122. CrossRef Medline

Watson CS, Wroton HW, Kelly WJ, Benbassat CA (1975) Factors in the discrimination of tonal patterns. I. Component frequency, temporal position, and silent intervals. J Acoust Soc Am 57:1175-1185. CrossRef Medline

Wickelgren WA (1969) Associative strength theory of recognition memory for pitch. J Math Psychol 61:13-61.

Wilsch A, Obleser J (2016) What works in auditory working memory? A neural oscillations perspective. Brain Res 1640:193-207. CrossRef Medline

Wilsch A, Henry MJ, Herrmann B, Maess B, Obleser J (2015) Alpha oscillatory dynamics index temporal expectation benefits in working memory. Cereb Cortex 25:1938-1946. CrossRef Medline

Wöstmann M, Herrmann B, Wilsch A, Obleser J (2015) Neural alpha dynamics in younger and older listeners reflect acoustic challenges and predictive benefits. J Neurosci 35:1458-1467. CrossRef Medline

Wöstmann M, Lim SJ, Obleser J (2017) The human neural alpha response to speech is a proxy of attentional control. Cereb Cortex 27:3307-3317. CrossRef Medline 